\title{
Image Diagnosis: Iliopsoas Abscess from Crohn Disease
}

Ashley S Abraham; Michelle Y Liu; David R Vinson, MD

E-pub: 05/18/2017

\section{CASE PRESENTATION}

A 42-year-old woman presented to her primary care physician with several years of intermittent right-sided lower abdominal cramps and pain associated with diarrhea, which during the previous 3 weeks had worsened in frequency and severity. Her physician ordered a contrast-enhanced abdominal computed tomography (CT) scan, which revealed terminal ileitis, the differential of which included Crohn disease as well as infectious and neoplastic processes. An inflammatory bowel disease serology panel showed a pattern consistent with Crohn disease. The patient was started on oral mesalamine. Outpatient colonoscopy showed the ileocecal valve to be narrowed with evidence of erythema, friable mucosa, and beefy ileal mucosa. Biopsies revealed ulcerations and granulation tissue.

During the next 2 weeks, the patient's abdominal pain failed to subside, so she was started on a 12-day tapered course of oral prednisone. During the last 4 days of her steroid regimen, she developed new-onset right hip pain and weakness of hip flexion, causing a moderate gait disturbance. She presented to the Emergency Department for evaluation and reported an increase in her mild, long-standing, right lower quadrant abdominal pain. She denied trauma, nausea, vomiting, fever, chills, or bloody stool, and had no history of abdominal surgery or appendicitis.

On physical examination, the patient was afebrile and had minimal right lower quadrant abdominal tenderness without guarding or rebound. She had moderate weakness of right hip flexion with limited range of motion. Her white blood cell count was elevated at $15.110^{9} / \mathrm{L}$. The rest of her complete blood cell count was normal, as were her electrolytes, creatinine, liver function tests, urinalysis, and pregnancy test. The emergency physician sought to exclude bony pathology and so ordered x-rays of the right hip, which were unremarkable. A contrast-enhanced CT scan of the abdomen revealed a 5-cm right iliopsoas abscess containing air. The terminal ileum was thick-walled with pericolonic inflammatory changes. The dilated adjacent bowel was consistent with local ileus (Figure 1).

The patient was started on intravenous piperacillin/tazobactam and admitted to the hospital. Symptoms improved the next day after CT-guided percutaneous abscess drainage, the culture of which was positive for Klebsiella pneumoniae. This common gram-negative enteric organism was sensitive to the administered antibiotics. The patient was discharged home on hospital day

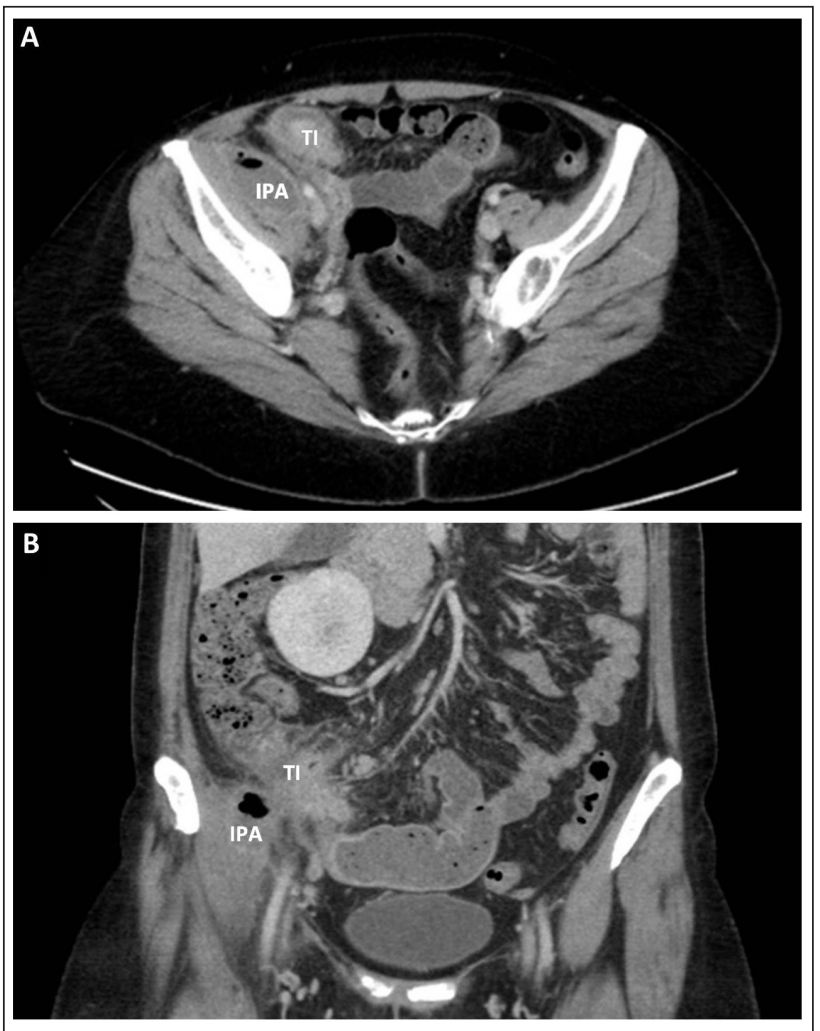

Figure 1. Axial view (A) and coronal view (B) contrast-enhanced computed tomography scans of the abdomen showing the terminal ileum (TI) and iliopsoas abscess (IPA).

four with a seven-day course of oral amoxicillin/clavulanate and ciprofloxacin. Her drain was removed one week later.

One month after the initial presentation, the patient returned to the Emergency Department with increasing right lower quadrant abdominal pain. A recurrent abscess was seen on contrastenhanced abdominal CT. She underwent exploratory laparotomy with wash out of the abscess and drain placement. Acute appendicitis was also discovered and removed. The affected bowel was resected. A communication between the bowel and the abscess was not described. The pathology report noted chronic active

Ashley S Abraham is a Research Intern with the CREST (Clinical Research on Emergency Services and Treatment) Network in Sacramento, CA. E-mail: ashley.sa.abraham@gmail.com. Michelle Y Liu is a Research Assistant with the CREST (Clinical Research on Emergency Services and Treatment) Network in Oakland, CA. E-mail: myxliu@berkeley.edu.

David R Vinson, MD, is a Senior Emergency Physician at the Sacramento Medical Center and Co-Chair of the CREST (Clinical Research on Emergency Services and Treatment) Network. He is an Adjunct Investigator at the Division of Research in Oakland, CA, and an Assistant Clinical Professor, Volunteer Clinical Faculty, in the Department of Emergency Medicine at the University of California, Davis in Sacramento. E-mail: drvinson@ucdavis.edu. 
ileitis with ulceration, granulation tissue, and transmural inflammation. No obvious perforation was identified on the appendix. Cultures from the abscess were negative. During the following 12 months, the patient was stable on mesalamine and experienced only 1 exacerbation requiring brief steroid treatment. She developed no further abscesses.

\section{DISCUSSION}

Crohn disease is a common cause of secondary iliopsoas abscesses, occurring in an estimated $0.4 \%$ to $4.3 \%$ of patients. ${ }^{1-3}$ Although long-term corticosteroid use with its attendant immunosuppression could increase the risk for abscess formation, a short course is unlikely to have the same effect. ${ }^{4-6}$ The diagnosis of iliopsoas abscess can be difficult to make, and delays in diagnosis can result in sepsis and organ failure and are known to increase mortality. ${ }^{2,3,7}$ Hip pain with impaired flexion and antalgic gait are characteristic complaints associated with iliopsoas abscesses and can serve as diagnostic clues. The pain, though usually localized to the hip, may radiate up into the flank area or down into the thigh. ${ }^{1,2,8-10}$ Clinicians should keep this diagnosis in mind when evaluating hip complaints in a patient with Crohn disease.

Crohn disease increases the risk for contiguous abscesses because the transmucosal inflammation predisposes patients to bowel wall perforations and fistula formation. ${ }^{11}$ Gram-negative bacilli and anaerobes are the most common pathogens identified in intestinal-associated abscesses..$^{10}$ Kpneumoniae, identified in our patient's iliopsoas abscess, has been associated with more invasive infections. ${ }^{12}$

Apart from removal of the inflamed bowel, patients with Crohn disease and a treated first abscess are at risk for local abscess recurrence. Treatment of an iliopsoas abscess requires drainage and antibiotic therapy, but recurrent abscesses may necessitate surgical resection of the affected bowel, as in our case.

\section{Disclosure Statement}

The author(s) have no conflicts of interest to disclose.

\section{How to Cite this Article}

Abraham AS, Liu MY, Vinson DR. Image diagnosis: lliopsoas abscess from Crohn disease. Perm J 2017;21:16-150. DOl: https://doi.org/10.7812/TPP/16-150.

\section{Acknowledgement}

We are grateful to Vignesh Arasu, MD, University of California, San Francisco School of Medicine, for his radiologic expertise.

\section{References}

1. Ogihara M, Masaki T, Watanabe T, et al. Psoas abscess complicating Crohn's disease: Report of a case. Surg Today 2000;30(8):759-63. DOI: https://doi. org/10.1007/s005950070093.

2. Lobo DN, Dunn WK, Iftikhar SY, Scholefield JH. Psoas abscesses complicating colonic disease: Imaging and therapy. Ann R Coll Surg Engl 1998 Nov;80(6):405-9.

3. Mallick IH, Thoufeeq MH, Rajendran TP. lliopsoas abscesses. Postgrad Med J 2004 Aug;80(946):459-62. DOl: https://doi.org/10.1136/pgmj.2003.017665.

4. Alejbab F, Choonara I, Conroy S. Systematic review of the toxicity of short-course oral corticosteroids in children. Arch Dis Child 2016 Apr;101(4):365-70. DOI: htps:I/ doi.org/10.1136/archdischild-2015-309522.

5. Walters JA, Tan DJ, White CJ, et al. Systemic corticosteroids for acute exacerbations of chronic obstructive pulmonary disease. Cochrane Database Syst Rev 2014 Sep 1;(9):CD001288. DOl: https://doi.org/10.1002/14651858.CD001288.pub4.

6. Aberdein J, Singer M. Clinical review: A systematic review of corticosteroid use in infections. Crit Care 2006 Feb;10(1):203. DOl: https://doi.org/10.1186/cc3904.

7. Tabrizian P, Nguyen SQ, Greenstein A, Rajhbeharrysingh U, Divino CM. Management and treatment of iliopsoas abscess. Arch Surg 2009 Oct;144(10):946-9. DOI: https:/l doi.org/10.1001/archsurg.2009.144.

8. Sauer C, Gutgesell M. Ballet dancer with hip and groin pain: Crohn disease and psoas abscess. Clin Pediatr (Phila) 2005 Oct;44(8):731-3. DOl: https://doi. org/10.1177/000992280504400814.

9. Cargill T, Gupta V, Thomas P. Pyogenic iliopsoas abscess: An uncommon presentation of nonspecific leg pain. J Acute Med 2014 Dec;4(4):154-6. DOI: https:// doi.org/10.1016/i.jacme.2013.09.001.

10. Navarro López V, Ramos JM, Meseguer V, et al. Microbiology and outcome of iliopsoas abscess in 124 patients. Medicine (Baltimore) 2009 Mar;88(2):120-30. DOI: https://doi.org/10.1097/MD.0b013e31819d2748.

11. Jawhari A, Kamm MA, Ong C, Forbes A, Bartram Cl, Hawley PR. Intra-abdominal and pelvic abscess in Crohn's disease: Results of non-invasive and surgical management. Br J Surg 1998 Mar;85(3):367-371. DOI: https://doi.org/10.1046/j.13652168.1998.00575.x.

12. Cheng SP, Chang WW, Tsao YT. Gas-forming iliopsoas abscess: A Klebsiella pneumoniae-mediated invasive syndrome. J Emerg Med 2016 Nov;51(5):e127-e128. DOI: https://doi.org/10.1016/j.jemermed.2016.06.013.

\section{The Important Difference}

The most important difference between a good and indifferent clinician

lies in the amount of attention paid to the story of a patient.

— Sir Edward Farquhar Buzzard, 1st Baronet, KCVO, FRCP, British physician

and Regius Professor of Medicine at the University of Oxford 